\begin{tabular}{|c|c|}
\hline Title & Reactivity boundaries for chemical reactions associated with higher-index and multiple saddles \\
\hline Author(s) & Nagahata, Y utaka; Teramoto, Hiroshi; Li, Chun-Biu; Kawai, Shinnosuke; Komatsuzaki, Tamiki \\
\hline Citation & $\begin{array}{l}\text { Phy sical review. E, 88(4), 042923-1-042923-11 } \\
\text { https://doi.org/10.1103/Phy sRevE.88.042923 }\end{array}$ \\
\hline Issue Date & $2013-10-29$ \\
\hline Doc URL & http:/hdl.handle.net/2115/54549 \\
\hline Rights & @2013 A merican Physical Society \\
\hline Type & article \\
\hline File Information & PhysRevE_88_042923.pdf \\
\hline
\end{tabular}

Instructions for use 


\title{
Reactivity boundaries for chemical reactions associated with higher-index and multiple saddles
}

\author{
Yutaka Nagahata, ${ }^{1}$ Hiroshi Teramoto, ${ }^{1,2}$ Chun-Biu Li, ${ }^{2,3,4}$ Shinnosuke Kawai, ${ }^{1,2, *}$ and Tamiki Komatsuzaki ${ }^{1,2,4, \dagger}$ \\ ${ }^{1}$ Graduate School of Life Science, Hokkaido University, Kita 10, Nishi 8, Kita-ku, Sapporo 060-0810, Japan \\ ${ }^{2}$ Molecule and Life Nonlinear Sciences Laboratory, Research Institute for Electronic Science, Hokkaido University, \\ Kita 20 Nishi 10, Kita-ku, Sapporo 001-0020, Japan \\ ${ }^{3}$ Graduate School of Science, Department of Mathematics, Hokkaido University, Kita 10, Nishi 8, Kita-ku, Sapporo 060-0810, Japan \\ ${ }^{4}$ Research Center for Integrative Mathematics, Hokkaido University, Kita 12, Nishi 7, Kita-Ku, Sapporo, Hokkaido 060-8011, Japan
}

(Received 16 August 2013; published 29 October 2013)

\begin{abstract}
Reactivity boundaries that divide the origin and destination of trajectories are of crucial importance to reveal the mechanism of reactions, which was recently found to exist robustly even at high energies for index 1 saddles [Phys. Rev. Lett. 105, 048304 (2010)]. Here we revisit the concept of the reactivity boundary and propose a more general definition that can involve a single reaction associated with a bottleneck composed of higher-index saddles and/or several saddle points with different indices, where the normal form theory, based on expansion around a single stationary point, does not work. We numerically demonstrate the reactivity boundary by using a reduced model system of the $\mathrm{H}_{5}{ }^{+}$cation where the proton exchange reaction takes place through a bottleneck composed of two index 2 saddle points and two index 1 saddle points. The cross section of the reactivity boundary in the reactant region of the phase space reveals which initial conditions are effective in making the reaction happen and thus sheds light on the reaction mechanism.
\end{abstract}

DOI: 10.1103/PhysRevE.88.042923

PACS number(s): 05.45.-a, 34.10.+x, 45.20.Jj, 82.20.Db

\section{INTRODUCTION}

Studies of chemical reaction dynamics aim to understand how and why a system proceeds from its initial state to the final state in the process of reaction. Special interest lies in the question concerning which initial conditions make the reaction happen. Classically, the process of the chemical reaction can be regarded as motion of a point in the phase space propagating from a region corresponding to the reactant to another region corresponding to the product. Some phase-space points in the reactant region may go into the product region after time propagation, whereas other phase-space points stay in the reactant region without undergoing the reaction. Between these reactive initial conditions and nonreactive ones lies a boundary which we simply call here the reactivity boundary, which has previously been described as "boundary trajectories" [1-14] asymptotic to a periodic orbit dividing surface (pods) [7-14], a "boundary" of [1-14] reactivity bands [15-25], a "tube" [26], a "cylindrical manifold" [26], "impenetrable barriers" [27], a "stable or unstable manifold" of normally hyperbolic invariant manifolds (NHIM) [27], "reaction boundaries" [28], and also described on certain sections as "reactivity bands" [15-25], "reactivity map" [18-25], and "reactive island" [26]. The general definition of the reactivity boundary is the main subject of this paper.

The reactivity boundary is often discussed in relation to saddle points. A saddle point on a multidimensional potential energy surface is defined as a stationary point at which the Hessian matrix does not have zero eigenvalues and, at least, one of the eigenvalues is negative. Saddle points are classified

\footnotetext{
*Present address: Department of Chemistry, Faculty of Science, Shizuoka University, 836 Ohya, Suruga-ku, Shizuoka 422-8529, Japan.

†tamiki@es.hokudai.ac.jp
}

by the number of negative eigenvalues and a saddle that has $n$ negative eigenvalues is called an index- $n$ saddle. In particular, the index 1 saddle on a potential surface has long been considered to make a bottleneck of reactions [29,30], with the sole unstable direction corresponding to the "reaction coordinate." This is because the index 1 saddle is considered to be the lowest energy stationary point connecting two potential minima, of which one corresponds to the reactant and the other to the product, and the trajectory must traverse the vicinity of the index 1 saddle from the reactant to the product [31-33].

Such reactivity boundaries have been investigated from early period of the study of reaction dynamics. Especially reactivity boundaries of atom-diatom reactions were extensively studied by Wright et al. [18-23] and Pechukas et al. [1-14]. Wigner introduced the asymptotic reactant and product regions to calculate the reaction rate in his transition state theory [34]. Independently, Wright et al. showed reactive bands, which had been found by Wall et al. [15-17], in the reactivity maps of $\mathrm{H}+\mathrm{H}_{2}$ and its isotopic variants [18-23] that consist of bands of nonreactive regions and reactive regions of each product. The approach was initiated by Ref. [18] to see the origin of continuous shift of peak in graph of initial relative translational (kinetic) energy versus time spent in "reaction shell" for given initial vibrational phase angles. After Ref. [18], a series of studies was reported for collinear (1D) [19], isotope [20], and coplanar (2D) [22] reactions and a 3D [23] reaction and also on an improved potential energy surface [21] with initial relative translational energy versus initial phase angle $\theta$. Chesnavich et al. observed boundary trajectories of the collision-induced dissociation of the $\mathrm{H}+\mathrm{H}_{2}$ reaction $[24,25]$ that divide reactive $\left(\mathrm{H}_{2}+\mathrm{H}\right)$, nonreactive $\left(\mathrm{H}+\mathrm{H}_{2}\right)$ and dissociative $(\mathrm{H}+\mathrm{H}+\mathrm{H})$ regions in phase space.

Pechukas et al. revealed the role of a periodic orbit dividing surface in two-dimensional collinear atom-diatom reaction systems [1-14]. The importance of a periodic orbit around the 
interaction region was first recognized by Pechukas [1]. This series of research can be described by his words as follows:

Somewhere between these two trajectories is a "dividing" trajectory that falls away, neither to reactant nor to product; this is the required "vibration," across the saddle point region but not necessarily through the saddle point, and the curve executed on the plane by the vibration is the best transition state at that energy.

Pechukas and Pollak [2] and Sverdlik and Koeppl [3] observed such trajectories in the region of index 1 saddles of two-dimensional systems and recognized them as an "unstable invariant classical manifold" [11] and called them a periodic orbit dividing surface (pods) [8]. The pods can be identified as the best transition state [5] when there is only one pods at a given energy. Pechukas and Pollak investigated the advantage of pods against variational transition-state (TS) theory [4] and unified statistical theory [6]. They also revealed its role in the application of statistical theories to reaction dynamics $[6,7,9]$ and provided an iterative method to calculate reaction probability [10]. After the series of classical investigation they started to look at adiabatic motion perpendicular to pods [13] and quantum correspondence [12] and experimental correspondence [14] were elucidated. Those studies were mostly done on two degrees of freedom (DOF) systems. The problem of high dimensionality in the region of index 1 saddles was later overcome [26-28,35-54].

The dynamics around the saddle point have been recently investigated extensively in terms of nonlinear dynamics [26-28,35,38-54] in the context of TS theory [29,30] in molecular science. Among them, particularly relevant to the present work, is the finding of the "tube" [26] structures in phase space to conduct the reacting trajectories from the reactant to the product across an index 1 saddle. These studies revealed the firm theoretical ground for the robust existence of the reactivity boundaries emanating from the saddle region as well as the no-return TS in the phase space [50,51]. The scope of the dynamical reaction theory [51] is not limited to only chemical reactions but also includes, for example, ionization of a hydrogen atom under electromagnetic fields $[27,40]$, isomerization of clusters $[38,39]$, orbit designs in solar systems [55], and so forth. Recently, these approaches have been generalized to dissipative multidimensional Langevin equations $[41,43,44]$ based on a seminal work by Martens [53], laser-controlled chemical reactions with quantum effects $[45,46]$, and systems with rovibrational couplings $[47,48]$ and showed the robust existence of reactivity boundaries even while a no-return TS ceases to exist [28].

For complex systems, the potential energy surface becomes more complicated, and transitions from a potential basin to another involve not only index 1 saddles but also higher-index saddles [56-64]. Recently, the role of index 2 saddles revealed several dynamical aspects. For example, a simulation study on "phase transitions" from solidlike phase to liquidlike phase in a seven-atomic cluster [56] showed that trajectories spend more time in the region of higher-index saddles as the total energy of the system increases. Under the onset of "melting," its occupation ratio around the index 2 saddles correlates to Lindemann's $\delta$ and the configuration entropy that are well-known indices of phase transition. Another example is a systematical survey of global stability of the triangular Lagrange points L4 and L5 under the condition that the secondary mass $\mu$ is larger than the Gascheau's value $\mu_{G}$ (also known as the Routh value) in the restricted planar circular three-body problem [57]. Those Lagrange points become index 2 saddle points when the condition $\mu>\mu_{G}$ is met, and the range of $\mu$ was identified where the Lagrange points have global stability and periodic stable orbits around them.

Chemical reactions associated with index 2 saddles were also reported in several molecular systems [58-64] by using several searching algorithms (section 6.3, p. 298, of Ref. [65] and references therein). However, index 2 saddles have received much less attention than index 1 saddles. This may be because of the Murrell-Laidler theorem [66] that states the minimum energy path does not pass through any index higher than 1 saddle points if the second derivative of the Hamiltonian has nonzero eigenvalue at the saddle. Note, however, that it is not necessary for the highest energy point on the minimum energy path between two potential minima to be an index 1 saddle [67]. Moreover, one can still find many studies such as aminoborane [58], $\mathrm{PF}_{3}$ [58], $\mathrm{NH}_{5}$ [59], $\mathrm{NF}_{2} \mathrm{H}_{3}$ [60], water dimer $[61,62], \mathrm{H}_{5}{ }^{+}$[63], and $\mathrm{H}_{2} \mathrm{CO}$ [64] that identify a variety of index 2 saddles in molecular isomerization reactions.

A significant difference between reactions associated with a bottleneck made of an index 1 saddle and those through a higher-index saddle is that a single higher-index saddle does not necessarily serve as a bottleneck from one potential basin to another since index- $n(>1)$ saddles are almost always accompanied with saddles of an index less than $n$. Therefore, reactions associated with higher-index saddle(s) are dominated by a bottleneck composed of multiple saddles, as are its phasespace structures. This nonlocal property of the bottleneck is an essential difficulty in treating a reaction associated with higher-index saddles.

To reveal the fundamental mechanism of the passage through a saddle with an index greater than 1, the phase-space structure was recently studied on the basis of normal form (NF) theory [68-71]. For example, the pioneering studies to extend the dynamical reaction theory into higher-index saddles were reported [68] for concerted reactions. A dividing surface to separate the reactant and the product was proposed for higher-index saddles [69] and the associated phase-space structure was also discussed [70,71]. Those studies are based on NF theory and therefore rely on two assumptions. One is that no linear "resonance" is postulated between more than one reactive modes and the other is that the local dynamics around the index 2 or higher-index saddle plays a dominant role in determining the destinations and the origins of the trajectories. For the former assumption, Toda [72] addressed that linear resonance between two reactive modes may introduce a breakdown of the reactivity boundary. As for the latter assumption, Nagahata et al. [73] reported recently that the reactivity boundary extracted by use of the normal form does not necessarily give the barrier separating the reactivity in the original coordinate space for higher-index saddles.

Moreover, as described above, an index 2 saddle often coexists with index 1 saddles and therefore the reaction 
dynamics or the "bottleneck" should be determined through interplay among multiple saddle points. Additionally, the current theory for invariant manifolds that may dominate reactions associated with an index 2 saddle and a higher-index saddle are only for the largest repulsive direction [70,71]. However, Minyaev et al. [58], for example, showed that aminoborane has internal rotation associated with an index 2 saddle and index 1 saddles and that the weaker repulsive direction around the index 2 saddle, corresponding to the hindered internal rotation, connects the two minima.

Most studies for a reaction associated with higher-index saddles are based on NF, a perturbation theory around a single stationary point, and assume that NF can capture those reaction dynamics. To validate those studies, however, it is necessary, first, to clarify the concept of reactivity boundaries in reactions associated with a bottleneck composed of multiple saddles. The reactivity map [18-25] and Pechukas' foresight [1] are still important to generalize the concept to make it applicable when the reaction dynamics is not dominated by a single saddle point.

In the present paper, we first review the concept of reactivity boundaries for the linear system in Sec. II A. Then we generalize the concept to the reactions associated with a bottleneck possibly composed of multiple saddle points in Sec. II B. In Sec. III we demonstrate the numerical extraction of reactivity boundaries in a chemical system with a bottleneck composed of multiple saddle points, including both index 1 and index 2 saddles. The investigation reveals what initial condition should be prepared to make the reaction happen and why such initial conditions lead to reactions.

\section{THEORY}

In this section, we revisit the concept of reactivity boundaries developed previously (Sec. II A) and propose a more general definition that can involve a single reaction associated with a bottleneck composed of higher-index saddles and/or several saddle points with different indices, where the normal form theory, based on expansion around a single stationary point, does not work (Sec. II B).

\section{A. Linearized Hamiltonian}

In this subsection we review the concept of reactivity boundaries developed previously based on the theory of dynamical systems [26-28,35,38-54]. One of the simplest examples of reactivity boundaries can be seen in the normal mode (NM) approximation. If the total energy of the system is just slightly above a stationary point, the $n$-DOFs Hamiltonian $H$ can well be approximated by a NM Hamiltonian $H_{0}$,

$$
H(\boldsymbol{p}, \boldsymbol{q}) \approx H_{0}(\boldsymbol{p}, \boldsymbol{q})=\sum_{j=1}^{n} \frac{1}{2}\left(p_{j}^{2}+k_{j} q_{j}^{2}\right)
$$

with NM coordinates $\boldsymbol{q}=\left(q_{1}, \ldots, q_{n}\right)$ and their conjugate momenta $\boldsymbol{p}=\left(p_{1}, \ldots, p_{n}\right)$, where $k_{j} \in \mathbb{R}$ is the "spring constant" or the curvature of the potential energy surface along the $j$ th direction. The constants $k_{j}$ can be positive or negative. If $k_{j}<0$, the potential energy is maximum along the $j$ th direction. Then the direction exhibits an unstable motion corresponding to "sliding down the barrier" and can

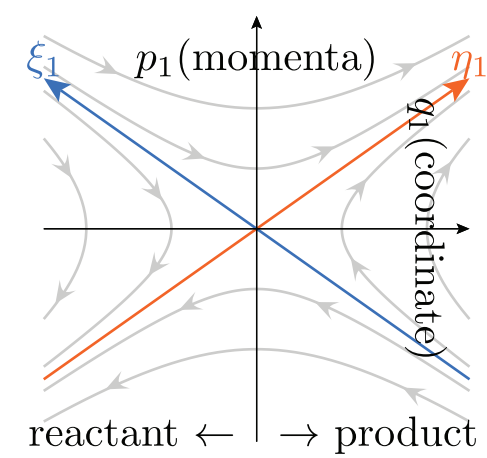

FIG. 1. (Color online) Phase-space flow of the normal mode with negative curvature (hyperbolic degree of freedom). Reactant and product are defined by the sign of $q_{1} . \eta_{1}=0$ (or $\xi_{1}$ axis) divides the destination of trajectories; Trajectories in $\eta_{1}>0$ go into the product side $\left(q_{1}>0\right)$ as $t \rightarrow+\infty$ and those in $\eta_{1}<0$ go into the reactant side $\left(q_{1}<0\right)$ ). Similarly, $\xi_{1}=0$ (or $\eta_{1}$ axis) divides the origin of trajectories; trajectories in $\xi_{1}>0$ originate from the reactant side and those in $\xi_{1}<0$ from the product side.

be regarded as "reaction coordinate." The index of the saddle corresponds to the number of negative $k_{j}$ 's. Phase-space flow of the DOF with negative $k_{j}$ is depicted in Fig. 1. Here one can introduce the following coordinates:

$$
\eta_{j}=\left(p_{j}+\lambda_{j} q_{j}\right) /\left(\lambda_{j} \sqrt{2}\right), \quad \xi_{j}=\left(p_{j}-\lambda_{j} q_{j}\right) / \sqrt{2},
$$

corresponding to eigenvectors of the coefficient matrix of the linear differential equation [Eq. (1)] with eigenvalue $\lambda_{j}= \pm \sqrt{-k_{j}}$. Here one can also introduce another set of coordinates,

$$
I_{j}=\xi_{j} \eta_{j}, \quad \theta_{j}=\ln \left|\lambda_{j} \eta_{j} / \xi_{j}\right| / 2,
$$

called the "action" and "angle" variables. When Eq.(1) holds, the action variable is an integral of motion, and trajectories run along the hyperbolas given by $I_{j}=$ const., shown by the gray lines in Fig. 1. The $\eta_{j}$ and $\xi_{j}$ axes run along the asymptotic lines of the hyperbolas in Fig. 1. The Hamiltonian equation of motion can be written as

$$
\dot{\zeta}_{j} \approx-L_{H_{0}} \zeta_{j}=-\lambda_{j} L_{I_{j}} \zeta_{j}=\left(\begin{array}{cc}
-\lambda_{j} & 0 \\
0 & \lambda_{j}
\end{array}\right) \zeta_{j},
$$

where $\zeta_{j}=\left(\xi_{j}, \eta_{j}\right)^{\mathrm{T}}$ and the Lie derivative $L_{F}$ is defined as $L_{F} \boldsymbol{\zeta}_{k}=\left\{F, \zeta_{k}\right\}=\sum_{j=1}^{n}\left(\frac{\partial F}{\partial \eta_{j}} \frac{\partial \zeta_{k}}{\partial \xi_{j}}-\frac{\partial F}{\partial \xi_{j}} \frac{\partial \zeta_{k}}{\partial \eta_{j}}\right)$. One can tell the destination region and the origin region of trajectories from the signs of $\eta_{j}$ and $\xi_{j}$ as follows: If $\eta_{j}>0$, the trajectory goes into $q_{j}>0$ and, if $\eta_{j}<0$, then the trajectory goes into $q_{j}<0$. Therefore one can tell the destination of trajectories from the sign of $\eta_{j}$. Similarly, the origin of trajectories can be told from the sign of $\xi_{j}$. Hereafter we call the set $\eta_{j}=0$ "destination-dividing set" and $\xi_{j}=0$ "origin-dividing set," and each of these two sets constitute "reactivity boundaries."

When the NM picture dominates the dynamics around the stationary point, the form of Eq. (4) enables us to identify the fate of reaction. This is also generally the case if one can achieve a canonical transformation to turn the Hamiltonian into the form of $H=H(\boldsymbol{I})$, even though $\lambda_{j} \mathrm{~s}$ depend on initial $I_{j} \mathrm{~s}$.

This transformation has been mostly achieved by the normal form theory based on expansion around a single stationary point. The theory has been applied and developed 


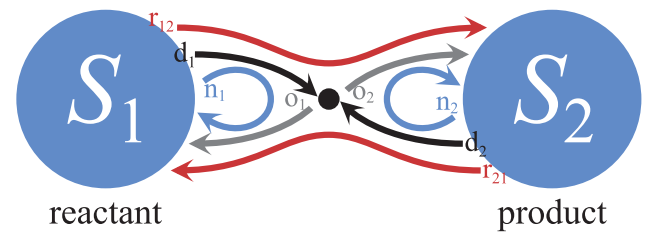

FIG. 2. (Color online) Blue large circles represent states $S_{1}$ and $S_{2}$. Arrows represent particular sorts of trajectories; blue arrows $\left(\mathrm{n}_{1}\right.$ and $\mathrm{n}_{2}$ ) represent nonreactive trajectories, while red ones $\left(\mathrm{r}_{12}\right.$ and $\left.r_{21}\right)$ represent reactive trajectories. Black arrows $\left(d_{1}\right.$ and $\left.d_{2}\right)$ and gray arrows $\left(\mathrm{o}_{1}\right.$ and $\left.\mathrm{o}_{2}\right)$ represent trajectories in the destination-dividing set, and those in the origin-dividing set, respectively.

to elucidate the mechanism of several reaction dynamics about a decade [27,28,38-51]. For practical applications the Lie canonical perturbation theory, developed by a Japanese astrophysicist, Gen-Ichiro Hori [74,75] (and equivalent theory was independently developed by Deprit $[76,77])$, has been frequently used.

\section{B. Reactivity boundary}

For complex molecular systems, the potential energy surface becomes more complicated, and a single transition from a potential basin to another involves not only index 1 saddles but also higher-index saddles. The normal form theory shown in Sec. II A, based on expansion around a single stationary point, may not work well for such complex systems, where the fate of the reaction may not be dominated solely by the local property of the potential around the point. Therefore the definition of the reactivity boundaries should not be based on perturbation theory. In this subsection, we seek for a more general definition of reactivity boundaries, so the definition can describe invariant objects previously studied (such as impenetrable barriers [27] and reactive island [26]), to analyze more complicated reactions by following the Pechukas' foresight [1].

A "state," which may refer to reactant or product, forms a certain region in the phase space $\Omega$. Let us denote the states by $S_{1}, \ldots, S_{N}$, which are disjoint subsets of $\Omega\left(S_{j} \subset \Omega\right.$ and $S_{i} \cap S_{j}=\emptyset$, where $i, j=1,2, \ldots, N$ and $\left.i \neq j\right)$.

Between the regions corresponding to the states, there can be an intermediate region $\Omega_{0}$ that does not belong to any of the states (Fig. 2): $\Omega=S_{1} \sqcup \cdots \sqcup S_{N} \sqcup \Omega_{0}$. Most of the trajectories in the intermediate region eventually go into either of the states as time proceeds. Likewise, when propagated backward in time, most of them turn out to originate from either of the states. Consider a set of trajectories that originate from state $S_{1}$ and go into state $S_{2}\left(\mathrm{r}_{12}\right.$ in Fig. 2) and another set consisting of trajectories that originate from $S_{1}$ and go back into the same state $S_{1}\left(\mathrm{n}_{1}\right.$ in Fig. 2). Between these two sets of trajectories there may lie a boundary which consists of trajectories that do not go into either of the states ( $d_{1}$ in Fig. 2). In the cases discussed in Sec. II A, such trajectories were seen to asymptotically approach into some invariant set(s) in the intermediate region. Suppose there exists such an invariant set $\Omega_{S}$, which is a codimension two subset of $\Omega_{0}$. We then consider codimension one subset $\Omega_{O D}, \Omega_{D D} \subset \Omega_{0}$ satisfying $\lim _{T \rightarrow-\infty} \phi^{T}\left(\Omega_{O D}\right)=\Omega_{S}$ and $\lim _{T \rightarrow+\infty} \phi^{T}\left(\Omega_{D D}\right)=\Omega_{S}$ where $\phi^{T}$ denotes time propagation, as follows:
(1) Destination-dividing set $\Omega_{D D}\left(\mathrm{~d}_{1}\right.$ and $\mathrm{d}_{2}$ in Fig. 2): A set of trajectories whose origin belongs to a certain state but whose destination does not belong to any state.

(2) Origin-dividing set $\Omega_{O D}\left(\mathrm{o}_{1}\right.$ and $\mathrm{o}_{2}$ in Fig. 2): A set of trajectories whose destination belongs to a certain state but whose origin does not belong to any state.

The former set constitutes a boundary dividing the destination regions of trajectories, whereas the latter constitutes a boundary dividing the origin regions of trajectories. The set of the trajectories (invariant set) that satisfy one of the above conditions will be called reactivity boundary in the following. The asymptotic limit $\Omega_{S}$ of the reactivity boundary, which belongs to neither reactant nor product, will be called the "seed" of the reactivity boundaries. The definition of the reactivity boundary (the destination- or the origin-dividing set) can be applied to systems with multiple states, since the definition of the reactivity boundaries are only based on a single state. This definition of the reactivity boundaries and their seed is a generalization of the previous invariant objects (the stable and unstable manifolds of NHIM, and the NHIM, respectively) studied in the literature [27,28,38-51] and summarized in Sec. II A.

\section{NUMERICAL DEMONSTRATIONS}

\section{A. Three DOFs model of $\mathbf{H}_{5}{ }^{+}$}

We demonstrate here a numerical calculation of the reactivity boundary defined in Sec. II with a model $\mathrm{H}_{5}{ }^{+}$system. This cation plays an important role in interstellar chemistry, especially because of the proton exchange reaction $\mathrm{H}_{3}{ }^{+}+$ $\mathrm{HD} \rightleftharpoons \mathrm{H}_{2}+\mathrm{H}_{2} \mathrm{D}^{+}$occurring through the $\mathrm{H}_{4} \mathrm{D}^{+}$intermediate. As shown in the previous ab initio calculation [63], the most stable structure of the $\mathrm{H}_{5}{ }^{+}$system is a weakly bound cluster of $\mathrm{H}_{2}$ and $\mathrm{H}_{3}{ }^{+}$moieties, with the $\mathrm{H}_{2}$ standing perpendicular to the $\mathrm{H}_{3}{ }^{+}$molecular plane. Being a multibody system, the $\mathrm{H}_{5}{ }^{+}$cation undergoes various isomerization reactions. Taking the four kinds of lowest stationary points (one minimum, two index 1 saddle points, and one index 2 saddle point), we have two reaction directions. One is a torsional isomerization where the $\mathrm{H}_{2}$ flips $180^{\circ}$ with the planar structure corresponding to the saddle point. The other is the proton exchange between the two moieties $\mathrm{H}_{2}+\mathrm{H}_{3}{ }^{+} \rightleftharpoons \mathrm{H}_{3}{ }^{+}+\mathrm{H}_{2}$.

In the present investigation, we treat the dynamics of $\mathrm{H}_{5}{ }^{+}$by confining it into a 3 DOF system. The dynamical variables are the center-of-mass distance $R$ between the two $\mathrm{H}_{2}$ moieties, the position $z$ of the central hydrogen atom along the center-of-mass axis, and the torsional angle $\varphi$ of the two $\mathrm{H}_{2}$ as shown in Fig. 3. The coordinate $z$ corresponds to the proton exchange reaction between the two moieties, while the angle $\varphi$ corresponds to the torsional isomerization. We

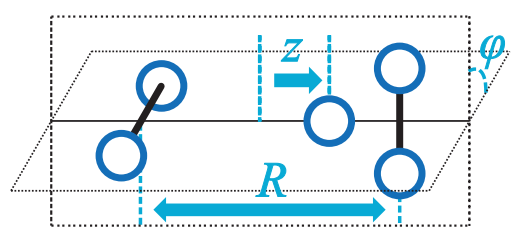

FIG. 3. (Color online) The $\mathrm{H}_{3}{ }^{+}+\mathrm{H}_{2} \rightarrow \mathrm{H}_{2}+\mathrm{H}_{3}{ }^{+}$reaction can be written with the three coordinates, $\varphi, R, z$, depicted in the figure. 
TABLE I. Structures and energies of four kinds of lowest stationary points of $\mathrm{H}_{5}{ }^{+}$. The energies are given relative to the first equilibrium point.

\begin{tabular}{ccccccc}
\hline \hline & $\varphi$ & $R / \AA$ & $z / \AA$ & Energy/cm & Ref. [63] & \\
\hline 1 & $\pi / 2$ & 2.18 & 0.19 & (Ref.) & (Ref.) & Global minimum \\
2 & $\pi / 2$ & 2.11 & 0 & 48.6 & 48.4 & Index 1 saddle \\
3 & 0 & 2.19 & 0.21 & 95.9 & 96.4 & Index 1 saddle \\
4 & 0 & 2.12 & 0 & 162.7 & 162.8 & Index 2 saddle \\
\hline \hline
\end{tabular}

calculated the potential energy surface at the $\operatorname{CCSD}(\mathrm{T})$ level, which is the same level with the previous calculation [63]. The ab initio calculations were performed at 439 points in the range $0 \leqslant|z| \leqslant 0.4 \AA$ and $2.09 \AA \leqslant R \leqslant 2.51 \AA$, with the $\mathrm{H}_{2}$ bond lengths optimized for each given value of $(z, R, \varphi)$. By checking the energy value, this region was confirmed to be sufficient to describe the motion with total energy below $200 \mathrm{~cm}^{-1}$. The potential energy values were then fitted to a cubic order polynomial in $\left(z^{2}, R, \cos 2 \varphi\right)$. The maximum fitting error was $0.8 \mathrm{~cm}^{-1}$, sufficiently small considering the total energy $170 \mathrm{~cm}^{-1}$ of the trajectories run in the present investigation. The structures and energies of the four lowest stationary points of the fitted surface are listed in Table I and compared with the literature values [63]. The mathematical expression of the fitted potential energy surface is available as the supporting information to this article.

We use this three-dimensional system as an illustrative model to demonstrate the concepts introduced in Sec. II. Note, however, that the real $\mathrm{H}_{5}{ }^{+}$system has larger DOFs (nine internal modes and three rotational modes). Quantum effects must also be considered for the complete treatment of this system. We here briefly mention that the concept of reactivity boundaries around the index 1 saddle point has recently been extended to incorporate ro-vibrational couplings $[47,48]$ and quantum effects $[45,46]$. It will be an important future work to combine these studies with the generalized reactivity boundaries proposed in the present paper. In the present numerical calculation we confine the system configuration into the three-dimensional subspace mentioned above for the sake of simplicity. We still note that the global minimum, the three lowest saddle points, and their unstable directions are all included in this subspace, while the motions transverse to this subspace are bath mode oscillations. This three-dimensional model is therefore expected to capture some of the essential properties of the isomerization and the proton exchange processes in the real $\mathrm{H}_{5}{ }^{+}$system with low energies.

There are two kinds of index 1 saddle points, denoted as 2 and $\mathbf{3}$, that correspond to the proton exchange and the torsional isomerization, respectively. The highest of these four kinds of stationary points are index 2 saddle points, denoted as $\mathbf{4}$, representing a concerted reaction of the proton exchange and the torsion. Figure 4 depicts the two-dimensional potential energy surface in $z$ and $\varphi$, where the $R$ is relaxed to the minimum energy for each given value of $(z, \varphi)$. There are four symmetrically equivalent points corresponding to the global minimum 1. Similarly, the saddle points 2,3 , and $\mathbf{4}$ have two, four, and two equivalent points, respectively.

The dynamical calculations of the present threedimensional model of $\mathrm{H}_{5}{ }^{+}$are performed by integrating the

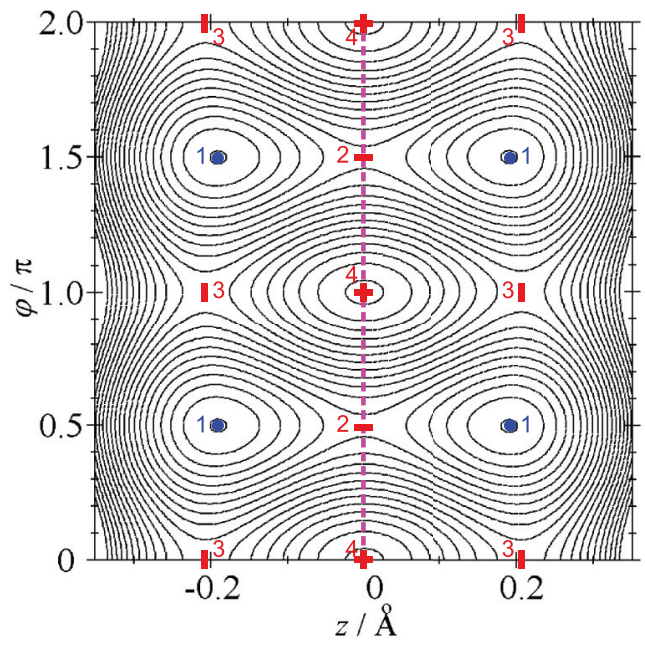

FIG. 4. (Color online) The potential energy surface as a function of $z$ and $\varphi$, representing the proton exchange and the torsional motion, where the other coordinate $R$ is optimized at each point $(z, \varphi)$. Each number corresponds to each stationary point listed in Table I. Blue points, red bars, and the red cross denote the potential minima, index 1 saddles, and the index 2 saddle, respectively. Contours are spaced with $10 \mathrm{~cm}^{-1}$. The initial condition for the calculation of the reactivity boundaries are prepared at $z=0$, where index 1 saddle points 2 and index 2 saddle points $\mathbf{4}$ are located (pink dashed line).

equation of motion given by the following Hamiltonian:

$$
H=\frac{1}{I_{\varphi}} p_{\varphi}^{2}+\frac{1}{2 \mu_{R}} p_{R}^{2}+\frac{1}{2 \mu_{z}} p_{z}^{2}+V(\varphi, R, z),
$$

where $p_{\varphi}$ is the angular-momentum conjugate to the torsional angle $\varphi$, and $p_{R}$ and $p_{z}$ are the linear momenta conjugate to $R$ and $z$, respectively. The reduced masses are

$$
\mu_{z}=\frac{4}{5} m_{\mathrm{H}}, \quad \mu_{R}=m_{\mathrm{H}},
$$

where $m_{\mathrm{H}}$ is the mass of the hydrogen atom and $I_{\varphi}$ is the moment of inertia of $\mathrm{H}_{2}$.

\section{B. Reactivity boundary in $\mathbf{H}_{5}{ }^{+}$}

As described in Sec. II B, the reactivity boundary typically consists of trajectories emanating from an invariant manifold in the intermediate region. It is calculated by propagating the phase-space points, either forward or backward in time, from the close vicinity of the invariant manifold. In the present investigation we focus on the proton exchange reaction from $\mathrm{H}_{2}+\mathrm{H}_{3}{ }^{+}$to $\mathrm{H}_{3}{ }^{+}+\mathrm{H}_{2}$ to demonstrate the extraction of reactivity boundary. The configuration $\mathrm{H}_{2}+\mathrm{H}_{3}{ }^{+}$corresponds to a region with $z>0$ and $\mathrm{H}_{3}{ }^{+}+\mathrm{H}_{2}$ with $z<0$. The intermediate region thus lies on some region around $z=0$. In this case the surface defined by $z=0$ and $p_{z}=0$ serves as an invariant manifold due to the symmetry of the system. This means that, once the phase-space point stays on that surface, it does perpetually irrespective of what values the other variables take. This invariant manifold is unstable in that any infinitesimally small deviation from the surface of $z=0$ and $p_{z}=0$ makes the phase-space point depart from the surface and fall down into one of the four well regions shown in Fig. 4. Therefore, the reactivity boundaries are stable and unstable manifolds of $z=0, p_{z}=0$ in this case. 

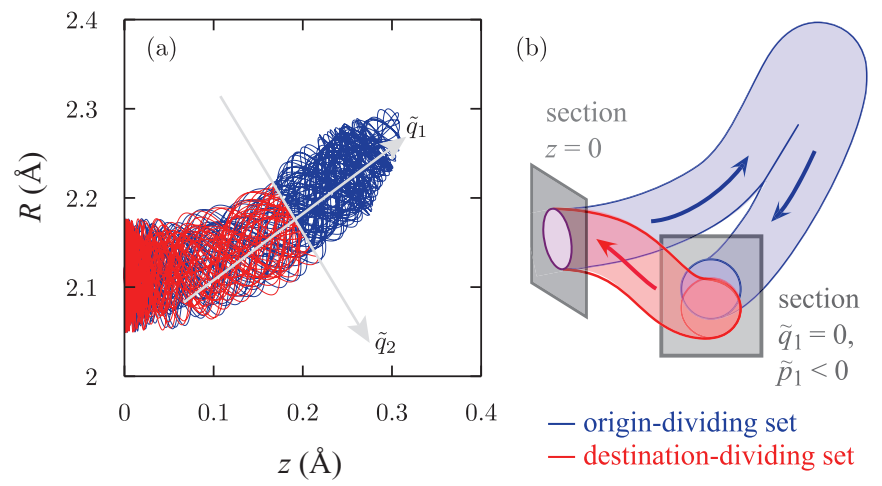

FIG. 5. (Color online) The reactivity boundaries of the $\mathrm{H}_{3}{ }^{+}+$ $\mathrm{H}_{2} \rightarrow \mathrm{H}_{2}+\mathrm{H}_{3}{ }^{+}$reaction. (a) Fifty randomly sampled trajectories from the destination-dividing set and the origin-dividing set, both constituting the reactivity boundaries, initiated from the section of $z=0$ and $p_{z} \simeq 0$ projected onto the $z-R$ space. The normal mode coordinates $\tilde{q}_{1}$ and $\tilde{q}_{2}$ at the potential minimum are shown by gray lines. (b) A schematic picture of reactivity boundaries depicted as "tubes" [26] departing from $z=0$ and $p_{z} \simeq 0$. Note here that the invariant manifold of $z=0$ and $p_{z}=0$ can involve multiple saddle points.

The extraction of reactivity boundary can be carried out as follows: We first uniformly sample phase-space points $\left(p_{z}=0, p_{R}, p_{\varphi}, z=0, R, \varphi\right)$ at a given total energy in that invariant manifold (see also the appendix for details). Second, we give each phase-space point a small positive deviation in $p_{z}$ and propagate it forward in time (corresponding to the origin-dividing set $\mathrm{o}_{2}$ in Fig. 2). Those trajectories correspond to the generalization of $\xi_{1}=0$ with positive $\eta_{1}$ to divide the origin of trajectories for normal mode approximation in Fig. 1. Likewise, the propagation of the phase-space points backward in time results in trajectories that divide the destination of trajectories, corresponding to the set $\mathrm{d}_{2}$ in Fig. 2 (Compare also with $\eta_{1}=0$ with negative $\xi_{1}$ for the normal mode Hamiltonian in Fig. 1). Note here again that the generalization involves two essential differences from the normal mode picture: One is the generalization to nonlinear Hamiltonian systems in which normal mode approximation does not hold, and the other is that the invariant manifold from which reactivity boundaries emanate can be associated not only with a single saddle point but with multiple saddle points with different indices.

Figure 5 shows 50 randomly chosen samples from the origin-dividing set and the destination-dividing set depicted on the $R-z$ space. The reactivity boundaries are only drawn until they first cross the section defined by $\tilde{q}_{1}=0$ and $\tilde{p}_{1}<0$ by the normal mode coordinate $\tilde{\boldsymbol{q}}$ and its conjugate momentum $\tilde{\boldsymbol{p}}$ at the potential minimum [the normal mode coordinates are shown by the gray arrows in Fig. 5(a)]. The reactivity boundaries are four-dimensional surfaces in an equienergy shell which divide reactive and nonreactive trajectories as schematically shown in Fig. 5(b).

Figures 6(a) and 6(b) show the origin-dividing set and the destination-dividing set on the $\tilde{q}_{1}=0, \tilde{p}_{1}<0$ section depicted by using 100000 trajectories whose initial conditions are uniformly sampled on the $z=0, p_{z} \simeq 0$ section (see also Appendix for details). Let us look into how reaction selectivity existing in the phase space can be rationalized or visualized in these projections. In Fig. 6(a), one can find few fingerprints of the reaction selectivity existing in the phase space with respect to the signs of the normal mode coordinate and momentum. The reaction path is curved in the $R-z$ space as shown in Fig. 5(a) and the saddle points exist on the negative side of $\tilde{q}_{2}$. Because Fig. 6(a) is the projection of the first intersections of the reactivity boundaries across the surface of $\tilde{q}_{1}=0$ and $\tilde{p}_{1}<0$ [i.e., all dots on Fig. 6(a) are moving towards the surface of $z=0$ ], one may expect that $\tilde{q}_{2}<0$ or $\tilde{p}_{2}<0$ on that surface should enhance the reaction probability, resulting in a nonuniform distribution of the reactivity boundaries on the $\tilde{p}_{2}-\tilde{q}_{2}$ space. However, as seen in Fig. 6(a), the reactivity boundaries are distributed rather uniformly in this space (e.g., no preference in the sign of $\tilde{p}_{2}$ ). This implies that preparing $\tilde{q}_{2}<0$ or $\tilde{p}_{2}<0$ on that surface does not increase the ability of the system to climb the reaction barrier. As shown in Fig. 5(a), the trajectories oscillate rapidly in the $\tilde{q}_{2}$ direction and the bath mode coordinate change its sign many times before coming close to $z=0$, where the saddle points $\mathbf{2}$ and $\mathbf{4}$ for the proton transfer reaction are located, while they slowly adapt to the curved reaction pathway. The dynamics near the index 1 and index 2 saddle points, thus, seems to be insensitive to the initial vibrational phase prepared in the well region.

Next let us turn to the $p_{\varphi}-\varphi$ projection in Fig. 6(b). The reactivity boundaries, both the destination-dividing set (red points in the figure) and the origin-dividing set, are confined in smaller values of $\left|p_{\varphi}\right|$ compared to energetically accessible values. This is because the energy is more distributed into the reactive mode when the momentum in the $\varphi$ direction is smaller. In contrast to the $\tilde{p}_{2}-\tilde{q}_{2}$ space, the reaction selectivity existing in the phase space manifests nonuniformity of the distribution of these reactivity boundaries in the $p_{\varphi}-\varphi$ space. The confinement of the destination-dividing set in smaller $\left|p_{\varphi}\right|$ is more pronounced in $\varphi \approx 0$ than in $\varphi \approx \pi / 2$, while the range of $\left|p_{\varphi}\right|$ of the origin-dividing set is more uniform in $\varphi$. Note that $\varphi=0$ corresponds to the planar configurations that involve both the index 1 saddle points 3 and the index 2 saddle points 4 (see Table I) and the reaction must proceed over the index 2 saddle when $\varphi \approx 0$ (Fig. 4). The relative barrier height through the index 2 saddle 4 for the proton transfer with $\varphi=0$ is $162.7-95.9=66.8 \mathrm{~cm}^{-1}$, which is higher than the barrier height through the index 1 saddle 2 with $\varphi=\pi / 2,48.6 \mathrm{~cm}^{-1}$ as seen from Table I. In order to put sufficient energy into the reactive mode to overcome the barrier, therefore, the momentum $p_{\varphi}$ in the $\varphi$ direction must be confined into much smaller values $\left|p_{\varphi}\right|$ for $\varphi \approx 0$ than for $\varphi \approx \pi / 2$ due to the conservation of total energy of the system. This interpretation, done by the relative barrier height with constant $\varphi$, is consistent with the plot of the sample trajectory (ds1) for small initial $\left|p_{\varphi}\right|$ in Fig. 7. This figure shows some representative sample trajectories in the $\varphi-z$ and $R-z$ spaces, whose locations in the $\tilde{p}_{2}-\tilde{q}_{2}$ and the $p_{\varphi}-\varphi$ spaces are also indicated as symbols in Figs. 6(a) and 6(b). It is seen that the motions along the reactive direction (approximately the $z$ direction) take place more rapidly than those along the $\varphi$ direction and the value of $\varphi$ does not change much during the course of the reaction.

On the other hand, the trajectories approaching the surface of $z=0$ and $p_{z}<0$ with large values of $\left|p_{\varphi}\right|$ at $\varphi \approx \pi / 2$ at the section correspond to the motion starting from the well region and approach to the index 2 saddle 4 , as shown in the $z-\varphi$ plane 

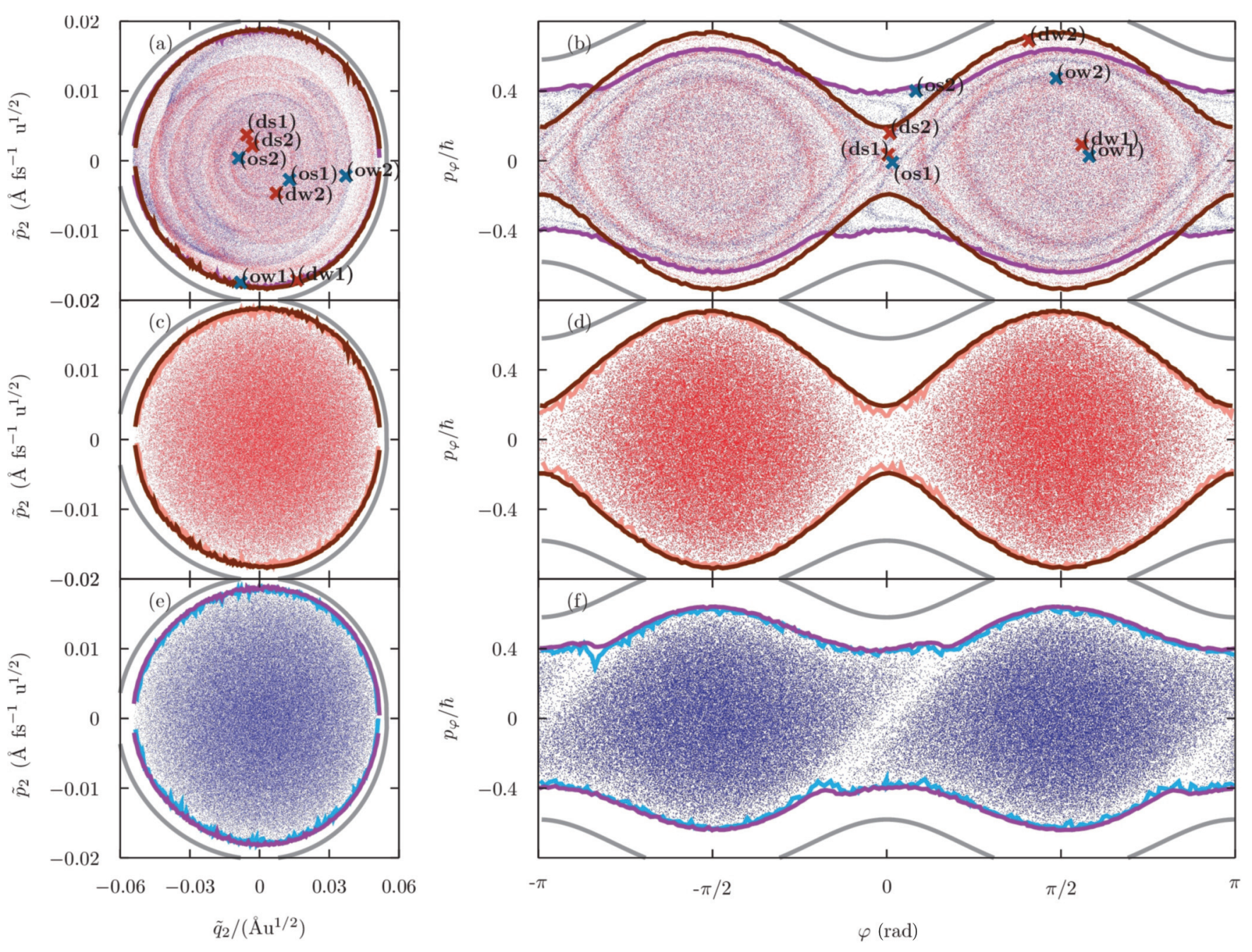

FIG. 6. (Color online) The reactivity of the $\mathrm{H}_{3}{ }^{+}+\mathrm{H}_{2} \rightarrow \mathrm{H}_{2}+\mathrm{H}_{3}{ }^{+}$reaction on the section of $\tilde{q}_{1}=0$, $\tilde{p}_{1}<0$. One hundred thousand trajectories are uniformly sampled on the surface of $z=0$ with positive momentum $p_{z} \simeq 0$ and evolved forward in time until they cross a surface defined by $\tilde{q}_{1}=0$ and $\tilde{p}_{1}<0$ by the normal mode coordinate $\tilde{\boldsymbol{q}}$ and its conjugate momentum $\tilde{\boldsymbol{p}}$ at the potential minimum (see also the appendix for details). The trajectories forming the origin-dividing set are shown by blue dots. Likewise, 100000 trajectories are similarly sampled on that surface with negative $p_{z} \simeq 0$ and propagated backward in time until they cross the surface. The trajectories forming the destination-dividing set are shown by red dots. [(a) and (b)] The projections of the first intersections of the destination-dividing set and the origin-dividing set crossing the surface of $\tilde{q}_{1}=0$ and $\tilde{p}_{1}<0$ on the section, respectively, onto the $\tilde{q}_{2}-\tilde{p}_{2}$ space and the $\varphi$ - $p_{\varphi}$ space. The gray lines denote the boundaries of energetically inaccessible region. The values are defined by the maximum and minimum of $p_{\varphi}$ at each $\varphi$. The cross symbols (dw1, dw2, etc.) represent the initial positions (on that plane) of the trajectories shown in Fig. 7. Brown (black) lines and purple (dark gray) lines are the maximum or minimum $\tilde{p}_{2}$ in (a) and the maximum or minimum $p_{\phi}$ in (b) of the reactivity boundaries (dots) respectively. [(c), (d), (e), and (f)] The projections of the phase-space points that are going into the product side [(c) and (d)] and those that have come from the product side [(e) and (f)] are depicted to conform "inside" of reactivity boundaries and to check validity of the extraction of the reactivity boundaries. Orange (gray) lines in (c) and cyan (gray) lines in (e) are the maximum or minimum $\tilde{p}_{2}$ of the sets of the reactive points. Brown (black) lines in (c) and purple (dark gray) lines in (e) are the maximum or minimum $\tilde{p}_{2}$ of the reactivity boundaries. Similarly, Orange (gray) lines in (d) and cyan (gray) lines in (f) are the maximum or minimum $p_{\varphi}$ of the sets of the reactive points. Brown (black) lines in (d) and purple (dark gray) lines in (f) are the maximum or minimum $p_{\varphi}$ of the reactivity boundaries.

in Fig. 7 (dw2). This is in contrast with the trajectories starting with small $\left|p_{\varphi}\right|$ at $\varphi \approx \pi / 2$ and approaching the index 1 saddle $2(\mathrm{dw} 1)$. If we regard $\left(p_{\varphi}, \varphi\right)$ as roughly corresponding to the nonreactive mode, this situation seems to be counterintuitive in that when the nonreactive degree of freedom is more excited (i.e., larger $\left|p_{\varphi}\right|$ ) the system is more likely to approach the higher-index saddle with a larger barrier height. This arises from the fact that the "reaction direction" for proton transfer through the index 2 saddle is not simply along $z$ but runs diagonal in the $z-\varphi$ plane as the system goes from the well directly to the index 2 saddle 4 . The large momentum $\left|p_{\varphi}\right|$ is also used for approaching the higher barrier of the index 2 saddle $\mathbf{4}$ and, therefore, the large initial value $\left|p_{\varphi}\right|$ is favored for the reaction over the index 2 saddle. All the above discussions explain the nonuniformity of the range of $p_{\varphi}$ with respect to $\varphi$ for the destination-dividing set in Fig. 6(b).

Compared to the destination-dividing set, the origindividing set is more uniformly distributed along $\varphi$ [see blue 

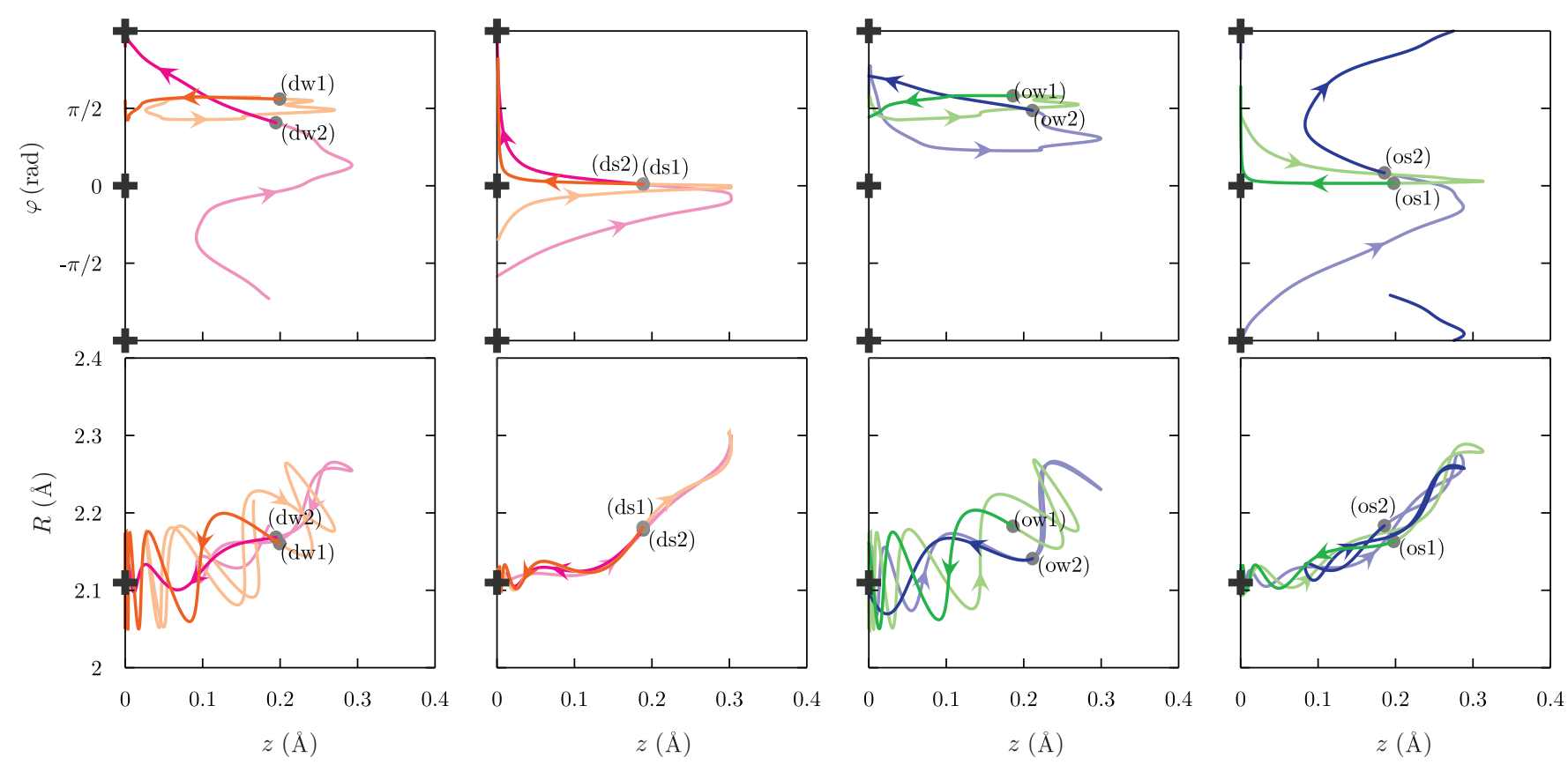

FIG. 7. (Color online) The representative sample trajectories forming the reactivity boundaries in Figs. 6(a) and 6(b) on the $\varphi-z$ space and the $R-z$ space. The gray points denote the locations in these spaces when those sample trajectories intersect the section of $\tilde{q}_{1}=0$ with $\tilde{p}_{1}<0$. The symbol + denotes the location of the index 1 point $\mathbf{2}$ or index 2 saddle point 4 . The difference of the location of the two saddle points is invisible in the $R-z$ projection with this resolution. The magenta and orange trajectories are of the destination-dividing set. The blue and green trajectories are of the origin-dividing set. The color grade represents the time course of trajectories obeying the Hamiltonian: Time goes from the light to the dark grade, and the light and dark correspond to before and after the intersection of the section of $\tilde{q}_{1}=0$ with $\tilde{p}_{1}<0$. For instance, trajectory (dw2) indicates that of the destination-dividing set in the well region with "large" $\left|p_{\varphi}\right|$. Trajectory (os1) indicates that of the origin-dividing set at the index $1 s$ addle region with "small" $\left|p_{\varphi}\right|$.

dots in Fig. 6(b)]. This arises from the choice of the cross section for observing the reactivity boundaries. We chose the section of $\tilde{q}_{1}=0$ with $\tilde{p}_{1}<0$ that is located at the potential minimum. With this choice, we are observing the origin-dividing set after it is bounced by the potential wall in the large- $z$ region [Fig. 5(a)]. As seen in the sample trajectories (os1), (os2), (ow1), and (ow2) in Fig. 7, the value of $\varphi$ changes during the stay in the well region. The change of $p_{\varphi}$ due to the energy exchange between the $\varphi$ mode and the others can also be seen by the direction of the trajectories. Therefore the longer time between the preparation (at $z=0$ ) and the observation $\left(\tilde{q}_{1}=0\right.$ with $\left.\tilde{p}_{1}<0\right)$ of the destination-dividing set than the origin-dividing set causes some further "mixing" in $\left(\varphi, p_{\varphi}\right)$ and the reaction selectivity is lost compared to the direct cross section as observed for the destination-dividing set in Fig. 6.

Reactivity boundaries are four-dimensional objects, and we cannot capture their full characteristics by the two-dimensional projections. In contrast to normal mode approximation or normal form theory locally expanded in the vicinity of a single saddle point, for our purposes, the analytic formula of the underlying reaction coordinate is hard to derive and the invariant manifold locally extracted in the vicinity of a single point or a collection of multiple saddle points with different indices might not necessarily provide the boundary to divide the fates of the reactions originated from the well region far from the saddles [73].
To check the validity of our numerical extraction of reactivity boundaries, we note the fact that both reactive and nonreactive trajectories must exist in the vicinity of the reactivity boundaries. We therefore check the reactivity of trajectories in the vicinity of each sampled point $\left(\tilde{p}_{2}, \tilde{q}_{2}, \varphi, p_{\varphi}\right)$ on the reactivity boundaries on the section. Sampling was made of phase -space points $\left(\tilde{p}_{2}^{\prime}, \tilde{q}_{2}^{\prime}, \varphi^{\prime}, p_{\varphi}^{\prime}\right)$ that satisfy

$$
\begin{aligned}
& \left|\frac{\tilde{p}_{2}^{\prime}-\tilde{p}_{2}}{0.02 \AA \mathrm{Au}^{1 / 2} \mathrm{fs}^{-1}}\right|^{2}+\left|\frac{\tilde{q}_{2}^{\prime}-\tilde{q}_{2}}{0.06 \AA \mathrm{u}^{1 / 2}}\right|^{2} \\
& +\left|\frac{\varphi^{\prime}-\varphi}{\pi}\right|^{2}+\left|\frac{p_{\varphi}^{\prime}-p_{\varphi}}{0.8 \hbar}\right|^{2}=10^{-20}
\end{aligned}
$$

for all the sampled points $\left(\tilde{p}_{2}, \tilde{q}_{2}, \varphi, p_{\varphi}\right)$ of the reactivity boundaries. As expected, both reactive and nonreactive trajectories were found from this sampling (data not shown).

To give more visual representation for the validity of our numerical extraction of reactivity boundaries, we uniformly sampled 1000000 points on the $\tilde{q}_{1}=0, \tilde{p}_{1}<0$ section in the well region and propagated them forward and backward in time. The phase-space points that turned out to go into the other well region in the forward time propagation are shown in Figs. 6(c) and 6(d) by projection on the $\tilde{q}_{2}-\tilde{p}_{2}$ space and the $\varphi-p_{\varphi}$ space. Those that turned out to have come from the other well in the backward propagation are shown in Figs. 6(e) and $6(\mathrm{f})$. Of the total 1000000 sampled points, about 100000 were found to be reactive trajectories. 
As can be seen in Figs. 6(c)-6(f), a good coincidence was observed in the maximum or minimum $\tilde{p}_{2}$ and $p_{\varphi}$ at each $\tilde{q}_{2}$ and $\varphi$ between the reactive trajectories [corresponding to the inside of "tubes" [26] in Fig. 5(b)] and the reactivity boundaries. In any neighborhood of the reactivity boundaries extracted from the surface of $z=0$ and $p_{z} \simeq 0$ apart from the well regions, reactive trajectories exist in the projected space. The results, therefore, also give some support (necessary condition) to the validity of the reactivity boundaries calculated in the present investigation.

\section{CONCLUSION AND PERSPECTIVES}

In this article, the concept of reactivity boundary, which is an invariant manifold lying between reacting and nonreacting trajectories in the phase space, was revisited and generalized. It is defined as a set of trajectories that converge into a seed of reactivity boundaries. The latter is located between the reactant and the product regions and goes neither into the reactant or the product, in either forward or backward time propagation. When only one saddle point controls the reaction dynamics and the energy is not very high above the saddle point, the reactivity boundaries are readily extracted analytically by normal form theory. The definition given here is, however, not limited to such cases but generalized to a single reaction passing through multiple saddle points including higher-index saddles.

The reactivity boundaries constitute a skeleton of the phase space of the reaction system. Observation of their locations in certain cross sections tells us which initial conditions can lead to chemical reactions. We applied the concept of the reactivity boundaries to the three-dimensional model system of the proton exchange reaction associated with a bottleneck composed of two index 1 saddles (2) and two index 2 saddles (4) in the $\mathrm{H}_{5}{ }^{+}$cation. The bath mode vibration represented by the normal mode $\left(\tilde{p}_{2}, \tilde{q}_{2}\right)$ was found to be almost separate from the reactive mode, and the fast change of its vibrational phase masked the reaction selectivity existing in the phase space.

On the other hand, the reaction selectivity in the phase space manifested a high degree of selectivity for the torsional motion, related to the existence of multiple types of saddle points for different values of the torsion angle. In addition to the reaction through the index 1 saddle $\mathbf{2}$ of the proton exchange, two limiting behaviors of reacting trajectories were identified. In one group, the trajectories go from the index 1 saddle 3 of the torsion isomerization to the index 2 saddle 4. Small initial values of the torsional angular momentum $\left|p_{\varphi}\right|$ is favored for this reaction pathway because of the high energy difference between the index 2 saddle point 4 and the index 1 torsion saddle point 3 . The other group of the reacting trajectories is those going directly from the well region to the index 2 saddle 4 . For this group, high initial values of $\left|p_{\varphi}\right|$ are favored because the reaction pathway runs diagonal in the $z-\varphi$ plane rather than parallel to the $z$ direction. These pictures of the reaction dynamics were obtained with the help of the concept of reactivity boundaries stated in the present paper.

In this article we have focused on the first intersection of the reactivity boundaries across the section of $\tilde{q}_{1}=0$ with $\tilde{p}_{1}<0$ located in the well region. This corresponds to the fast stage of the reaction process, that is, "before leaving from that well" and "after entering with one reflection back by the potential wall in that well." Reactivity boundaries also enable us to quantify the slow stage of the process by the projection of the second, third, and fourth intersections of the boundaries onto, e.g., the $\varphi-p_{\varphi}$ space. Distributions of such intersections on some projected spaces can trace how statistical properties may emerge for slower time scales (yielding a more uniform distribution), making conventional statistical rate theories applicable. Note that, as demonstrated in this article, the first intersection corresponding to the reactive initial conditions are distributed in a nonuniform manner, to which conventional statistical rate theories are not applicable. The essential understanding of the reactions requires reactivity boundaries that enable us to predict the fate of reactions independent of which time scale is to be considered.

In the extraction scheme of reactivity boundaries presented in Sec. IIB, we have not restricted the definition of states to a local equilibrium state in which highly developed chaos is implicitly postulated. As known, at least for the 2-DOF systems in Refs. [78,79], there may exist several dynamic states within a single potential well whose number and reaction rate constants among them are energy dependent. The definition of states in Sec. II B can involve such dynamic states. In addition, as discussed in the text, the seed of reactivity boundaries existing between the states involves not necessarily only one single saddle point but also several saddle points with different indices.

However, there still remains the need for development of practical methods for extracting the reactivity boundaries. When only one saddle point plays a dominant role in determining the occurrence of the reaction, normal form theory readily extracts the seed of reactivity boundaries in an analytical way. In contrast, there is still no practical method applicable to general cases where more than one saddle point are involved in the reaction process [73].

In the present investigation, because of the existence of symmetry, we can identify the seed of reactivity boundaries in the intermediate region. What happens when such apparent symmetry does not exist, or is very difficult to find a priori, is still unresolved. It will be a challenge for future work to devise convenient methods to extract the seeds of reactivity boundaries and investigate how they look for nonsymmetric systems.

Also note that the seed of reactivity boundaries in our generalized definition is not necessarily a NHIM, hence, not necessarily structurally stable. This raises a question of how meaningful (robust) conclusions obtained for a particular system are, a problem to be addressed in future work.

\section{ACKNOWLEDGMENTS}

T.K. acknowledges Professor Takeshi Oka for many beneficial discussions. This work was partially supported by JSPS, Research Center for Computational Science, Okazaki, Japan; a Grant-in-Aid for Young Scientists (B) (to S.K.); a Grant-in-Aid for challenging Exploratory Research (to T.K.); and a Grant-in-Aid for Scientific Research (B) (to T.K.) from the Ministry of Education, Culture, Sports, Science and Technology. 


\section{APPENDIX: UNIFORM SAMPLING}

Here we explain how we sample the uniform distributions under constraints to depict the reactivity boundaries and the sets of reacted or reacting trajectories, i.e., those having just crossed the surface of $z=0$ from the product well and those being about to cross the surface, in the reactant well described in Sec. IIIB. To depict reactivity boundaries, we sample the position coordinate $(R, \varphi)$ according to the following distributions:

$$
\begin{aligned}
& \rho\left(R, \varphi ; z=0, p_{z}=0, H=E\right) \\
& \quad \propto \int \delta(E-H(\mathbf{p}, \mathbf{q})) \delta(z) \delta\left(p_{z}\right) d p_{R} d p_{z} d p_{\varphi} d z \\
& \quad \propto \sqrt{E-V(R, \varphi ; z=0)} .
\end{aligned}
$$

Here we define $\bar{\rho}_{\mathrm{sd}}(R, \varphi)=\sqrt{\frac{E-V(R, \varphi ; z=0)}{E-V_{0}}}$, yielding $0<$ $\bar{\rho}_{\text {sd }}<1$, where $V_{0}=\min _{R, \varphi} V(R, \varphi ; z=0)$. We employ the rejection method [80] to sample phase-space points with the distribution $\bar{\rho}_{\text {sd }}$. We first sample points uniformly in the range of $\varphi \in[-\pi, \pi]$ and $R \in[2 \AA, 2.2 \AA]$, which include the whole energetically accessible region. The point is accepted or rejected by the following criterion:

$$
\begin{cases}\text { accept } & \bar{\rho}_{\mathrm{sd}}(R, \varphi)>\mathrm{RAND}, \\ \text { reject } & \text { otherwise }\end{cases}
$$

where RAND is a uniform random number from 0 to 1 . Then we perform a sampling of the momentum for each sampled configuration as follows:

$$
\begin{aligned}
& p_{R}=\sqrt{2(E-V)} \sin \theta / m_{R}, \\
& p_{\varphi}=\sqrt{2(E-V)} \cos \theta /\left(I_{\varphi} / 2\right),
\end{aligned}
$$

where $\theta$ is a uniform random number from $-\pi$ to $\pi$.
Similarly, to depict the sets of reacted or reacting trajectories in the reactant well, we sample phase-space points according to the following distribution:

$$
\begin{aligned}
& \rho\left(\tilde{q}_{2}, \varphi ; \tilde{q}_{1}=0, \tilde{p}_{1}<0, H=E\right) \\
& \quad \propto \int \delta(E-H(\mathbf{p}, \mathbf{q})) \Theta\left(-\tilde{p}_{1}\right) \delta\left(\tilde{q}_{1}\right) d \tilde{p}_{1} d \tilde{p}_{2} d \tilde{p}_{\varphi} d \tilde{q}_{1} \\
& \quad \propto E-V\left(\tilde{q}_{2}, \varphi ; \tilde{q}_{1}=0\right) .
\end{aligned}
$$

Here $\Theta(x)$ is the Heaviside step function, and we define $\bar{\rho}_{\mathrm{wl}}\left(\tilde{q}_{2}, \varphi\right)=\left(E-V\left(\tilde{q}_{2}, \varphi ; \tilde{q}_{1}=0\right)\right) /\left(E-V_{0}\right)$, yielding $0<$ $\bar{\rho}_{\mathrm{wl}}<1$, where $V_{0}=\min _{\tilde{q}_{2}, \varphi} V\left(\tilde{q}_{2}, \varphi ; \tilde{q}_{1}=0\right)$. We sample points uniformly in the range of $\varphi \in[-\pi, \pi]$ and $\tilde{q}_{2} \in$ $\left[-0.15 \AA u^{1} / 2,0.15 \AA u^{1} / 2\right]$ which include the whole energetically accessible region on this section. We apply the same rejection method [80] to construct $\bar{\rho}_{\mathrm{wl}}$ distribution,

$$
\begin{cases}\text { accept } & \bar{\rho}_{\mathrm{wl}}\left(\tilde{q}_{2}, \varphi\right)>\mathrm{RAND}, \\ \text { reject } & \text { otherwise. }\end{cases}
$$

Then we perform a sampling of the momentum for each sampled configuration as follows:

$$
\begin{gathered}
\left\{\begin{array}{l}
\text { accept } \\
\text { reject }
\end{array} \begin{array}{l}
\sin \theta_{1}>\text { RAND, } \\
\text { otherwise }
\end{array}\right. \\
\tilde{p}_{\varphi}=\sqrt{2(E-V)} \sin \theta_{1} \sin \theta_{2}, \\
\tilde{p}_{1}=-\sqrt{2(E-V)} \cos \theta_{1}, \\
\tilde{p}_{2}=\sqrt{2(E-V)} \sin \theta_{1} \cos \theta_{2},
\end{gathered}
$$

since coordinate transformation to polar coordinates introduces phase-space Jacobian $J=2(E-V) \sin \theta_{1}$, where $\theta_{1}, \theta_{2}$ are uniform random numbers from 0 to $\pi / 2$ and from $-\pi$ to $\pi$, respectively.
[1] P. Pechukas, in Dynamics of Molecular Collisions Part B, Modern Theoretical Chemistry, edited by W. H. Miller (Plenum, New York, 1976), Chap. 6, pp. 269-322.

[2] P. Pechukas and E. Pollak, J. Chem. Phys. 67, 5976 (1977).

[3] D. Sverdlik and G. Koeppl, Chem. Phys. Lett. 59, 449 (1978).

[4] E. Pollak and P. Pechukas, J. Chem. Phys. 69, 1218 (1978).

[5] P. Pechukas and E. Pollak, J. Chem. Phys. 71, 2062 (1979).

[6] E. Pollak and P. Pechukas, J. Chem. Phys. 70, 325 (1979).

[7] M. S. Child and E. Pollak, J. Chem. Phys. 73, 4365 (1980).

[8] E. Pollak, M. S. Child, and P. Pechukas, J. Chem. Phys. 72, 1669 (1980).

[9] E. Pollak and R. D. Levine, J. Chem. Phys. 72, 2990 (1980).

[10] E. Pollak and M. S. Child, J. Chem. Phys. 73, 4373 (1980).

[11] P. Pechukas, Annu. Rev. Phys. Chem. 32, 159 (1981).

[12] E. Pollak, J. Chem. Phys. 74, 5586 (1981).

[13] E. Pollak, Chem. Phys. 61, 305 (1981).

[14] E. Pollak, Chem. Phys. Lett. 80, 45 (1981).

[15] F. T. Wall, L. A. Hiller, and J. Mazur, J. Chem. Phys. 29, 255 (1958).

[16] F. T. Wall, L. A. Hiller, and J. Mazur, J. Chem. Phys. 35, 1284 (1961).
[17] F. T. Wall and R. N. Porter, J. Chem. Phys. 39, 3112 (1963).

[18] J. S. Wright, G. Tan, K. J. Laidler, and J. E. Hulse, Chem. Phys. Lett. 30, 200 (1975).

[19] J. S. Wright, J. Chem. Phys. 64, 970 (1976).

[20] J. S. Wright and K. G. Tan, J. Chem. Phys. 66, 104 (1977).

[21] K. J. Laidler, K. Tan, and J. S. Wright, Chem. Phys. Lett. 46, 56 (1977).

[22] K. G. Tan, K. J. Laidler, and J. S. Wright, J. Chem. Phys. 67, 5883 (1977).

[23] J. S. Wright, J. Chem. Phys. 69, 720 (1978).

[24] B. K. Andrews and W. J. Chesnavich, Chem. Phys. Lett. 104, 24 (1984).

[25] M. E. Grice, B. K. Andrews, and W. J. Chesnavich, J. Chem. Phys. 87, 959 (1987).

[26] A. de Almeida, N. de Leon, M. A. Mehta, and C. Marston, Physica D 46, 265 (1990).

[27] S. Wiggins, L. Wiesenfeld, C. Jaffé, and T. Uzer, Phys. Rev. Lett. 86, 5478 (2001).

[28] S. Kawai and T. Komatsuzaki, Phys. Rev. Lett. 105, 048304 (2010). 
[29] S. Glasstone, K. J. Laidler, and H. Eyring, The Theory of Rate Processes (McGraw-Hill, New York, 1941).

[30] J. I. Steinfeld, J. S. Francisco, and W. L. Hase, Chemical Kinetics and Dynamics, 1st ed. (Prentice Hall, Upper Saddle River, NJ, 1989).

[31] J. Zhang, D. Dai, C. C. Wang, S. A. Harich, X. Wang, X. Yang, M. Gustafsson, and R. T. Skodje, Phys. Rev. Lett. 96, 093201 (2006).

[32] R. T. Skodje, D. Skouteris, D. E. Manolopoulos, S.-H. Lee, F. Dong, and K. Liu, Phys. Rev. Lett. 85, 1206 (2000).

[33] W. Shiu, J. J. Lin, and K. Liu, Phys. Rev. Lett. 92, 103201 (2004).

[34] E. Wigner, J. Chem. Phys. 5, 720 (1937).

[35] S. Wiggins, Physica D 44, 471 (1990).

[36] T. Komatsuzaki and M. Nagaoka, J. Chem. Phys. 105, 10838 (1996).

[37] T. Komatsuzaki and M. Nagaoka, Chem. Phys. Lett. 265, 91 (1997).

[38] T. Komatsuzaki and R. S. Berry, J. Chem. Phys. 110, 9160 (1999).

[39] T. Komatsuzaki and R. S. Berry, Proc. Natl. Acad. Sci. USA 98, 7666 (2001).

[40] T. Uzer, C. Jaffé, J. Palacián, P. Yanguas, and S. Wiggins, Nonlinearity 15, 957 (2002).

[41] T. Bartsch, R. Hernandez, and T. Uzer, Phys. Rev. Lett. 95, 058301 (2005).

[42] C.-B. Li, A. Shoujiguchi, M. Toda, and T. Komatsuzaki, Phys. Rev. Lett. 97, 028302 (2006).

[43] R. Hernandez, T. Bartsch, and T. Uzer, Chem. Phys. 370, 270 (2010).

[44] S. Kawai and T. Komatsuzaki, Phys. Chem. Chem. Phys. 13, 21217 (2011).

[45] H. Waalkens, R. Schubert, and S. Wiggins, Nonlinearity 21, R1 (2008).

[46] S. Kawai and T. Komatsuzaki, J. Chem. Phys. 134, 024317 (2011).

[47] S. Kawai and T. Komatsuzaki, J. Chem. Phys. 134, 084304 (2011).

[48] Ü. Çiftçi and H. Waalkens, Nonlinearity 25, 791 (2012).

[49] H. Teramoto, M. Toda, and T. Komatsuzaki, Phys. Rev. Lett. 106, 054101 (2011).

[50] M. Toda, T. Komatsuzaki, T. Konishi, R. S. Berry, and S. A. Rice, eds., Geometrical Structures of Phase Space in Multidimensional Chaos, Adv. Chem. Phys., Vols. 130A and 130B (John Wiley \& Sons, New York, 2005) and references therein.

[51] T. Komatsuzaki, R. S. Berry, and D. M. Leitner, eds., Advancing Theory for Kinetics and Dynamics of Complex, ManyDimensional Systems, Adv. Chem. Phys., Vol. 145 (John Wiley \& Sons, New York, 2011).

[52] C. Jaffé, J. Palacián, S. Kawai, P. Yanguas, and T. Uzer, Adv. Chem. Phys. 130A, 171 (2005).
[53] C. C. Martens, J. Chem. Phys. 116, 2516 (2002).

[54] T. Komatsuzaki and R. S. Berry, Adv. Chem. Phys. 123, 79 (2003).

[55] C. Jaffé, S. D. Ross, M. W. Lo, J. Marsden, D. Farrelly, and T. Uzer, Phys. Rev. Lett. 89, 011101 (2002).

[56] N. Shida, Adv. Chem. Phys. 130B, 129 (2005).

[57] B. Sicardy, Celest. Mech Dyn. Astr. 107, 145 (2010).

[58] R. M. Minyaev, D. J. Wales, and T. R. Walsh, J. Phys. Chem. A 101, 1384 (1997).

[59] R. M. Minyaev, I. V. Getmanskii, and W. Quapp, Russ. J. Phys. Chem. 78, 1494 (2004).

[60] I. V. Getmanskii and R. M. Minyaev, J. Struct. Chem. 49, 973 (2008).

[61] X. Huang, B. J. Braams, and J. M. Bowman, J. Phys. Chem. A 110, 445 (2006).

[62] A. Shank, Y. Wang, A. Kaledin, B. J. Braams, and J. M. Bowman, J. Chem. Phys. 130, 144314 (2009).

[63] Z. Xie, B. J. Braams, and J. M. Bowman, J. Chem. Phys. 122, 224307 (2005).

[64] J. M. Bowman and B. C. Shepler, Annu. Rev. Phys. Chem. 62, $531(2011)$.

[65] D. J. Wales, Energy Landscapes: Applications to Clusters, Biomolecules and Glasses, Cambridge Molecular Science (Cambridge University Press, Cambridge, UK, 2004).

[66] J. N. Murrell and K. J. Laidler, Trans. Faraday Soc. 64, 371 (1968).

[67] D. J. Wales and R. S. Berry, J. Chem. Soc., Faraday Trans. 88, 543 (1992).

[68] G. S. Ezra and S. Wiggins, J. Phys. A: Math. Theor. 42, 205101 (2009).

[69] P. Collins, G. S. Ezra, and S. Wiggins, J. Chem. Phys. 134, 244105 (2011).

[70] G. Haller, T. Uzer, J. Palacián, P. Yanguas, and C. Jaffé, Commun. Nonlinear Sci. Numer. Simul. 15, 48 (2010).

[71] G. Haller, T. Uzer, J. Palacián, P. Yanguas, and C. Jaffé, Nonlinearity 24, 527 (2011).

[72] M. Toda, AIP Conf. Proc. 245, 245 (2008).

[73] Y. Nagahata, H. Teramoto, C.-B. Li, S. Kawai, and T. Komatsuzaki, Phys. Rev. E 87, 062817 (2013).

[74] G.-i. Hori, Publ. Astron. Soc. Jpn. 18, 287 (1966).

[75] G.-i. Hori, Publ. Astron. Soc. Jpn. 19, 229 (1967).

[76] J. A. Campbell and W. H. Jefferys, Celestial Mech. 2, 467 (1970).

[77] A. Deprit, Celestial Mech. 1, 12 (1969).

[78] M. J. Davis and S. K. Gray, J. Chem. Phys. 84, 5389 (1986).

[79] R. Mackay, J. Meiss, and I. Percival, Physica D 13, 55 (1984).

[80] W. H. Press, S. A. Teukolsky, W. T. Vetterling, and B. P. Flannery, in Numerical Recipes: The Art of Scientific Computing, 3rd ed. (Cambridge University Press, Cambridge, UK, 2007), Chap. 7, p. 361 . 